\title{
THE ASSOCIATION BETWEEN CONTRAVERSIVE LATEROPULSION AND OUTCOMES POST STROKE: A SYSTEMATIC REVIEW
}

\author{
Jessica Nolan',2, Erin Godecke ${ }^{2,3,4}$, Barbara Singer ${ }^{2,5}$
}

PROSPERO ID: CRD42018115644

\section{Background}

Contraversive lateropulsion is common after stroke. Overall recovery of function, rehabilitation length of stay and discharge destination in those exhibiting lateropulsion may differ from stroke survivors without lateropulsion.

\section{Objectives}

1. Systematically review evidence for the association of presence and severity of lateropulsion after stroke with recovery of lateropulsion, functional outcome, rehabilitation length of stay, and discharge destination.

2. Appraise, analyse and report the findings of reviewed studies and to inform ongoing research.

\section{Methods}

Medline, CINAHL and Embase database searches were undertaken. Journal articles reporting on recovery of post-stroke lateropulsion, functional outcome, rehabilitation length of stay and / or discharge destination were included for review.

Search terms used:

MEDLINE

Search mode: Boolean / phrase

Stroke AND lateropulsion OR pusher syndrome OR pushing

Limits: English language

Publication type: journal article

CINAHL

Search mode: Boolean / phrase

Stroke AND lateropulsion OR pusher syndrome OR pushing

Limits: English language

Publication type: journal article

Embase

MeSH preferred term for stroke: cerebrovascular accident

Cerebrovascular accident AND lateropulsion OR pusher syndrome OR pushing

Limits: English language

Publication type: article

\section{Study Eligibility Criteria}

Journal articles published in English before 1 May 2019 reporting on recovery of post-stroke lateropulsion, functional outcome, rehabilitation length of stay and / or discharge destination were included for review. Single case reports were excluded.

\section{Data Sources}

Figure 1. PRISMA flow diagram

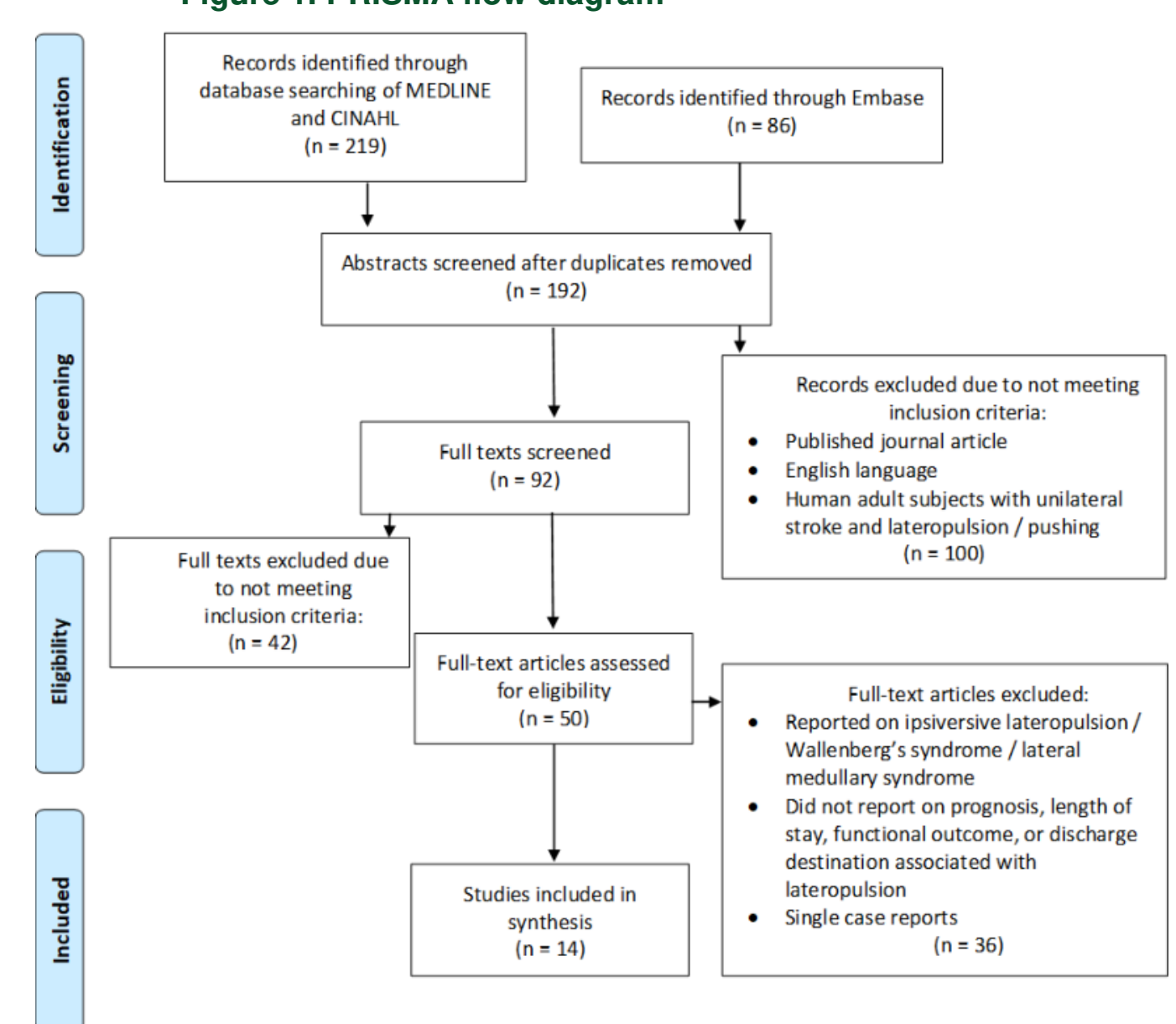

\section{Data Management}

Studies were assessed independently for inclusion by two authors, and discrepancies adjudicated by a third reviewer. Two assessors independently assessed risk of bias using the Risk of Bias Assessment Tool for Nonrandomized Studies (RoBANS) (Table 1) and size of cohort reported, and discrepancies were adjudicated by the third reviewer.

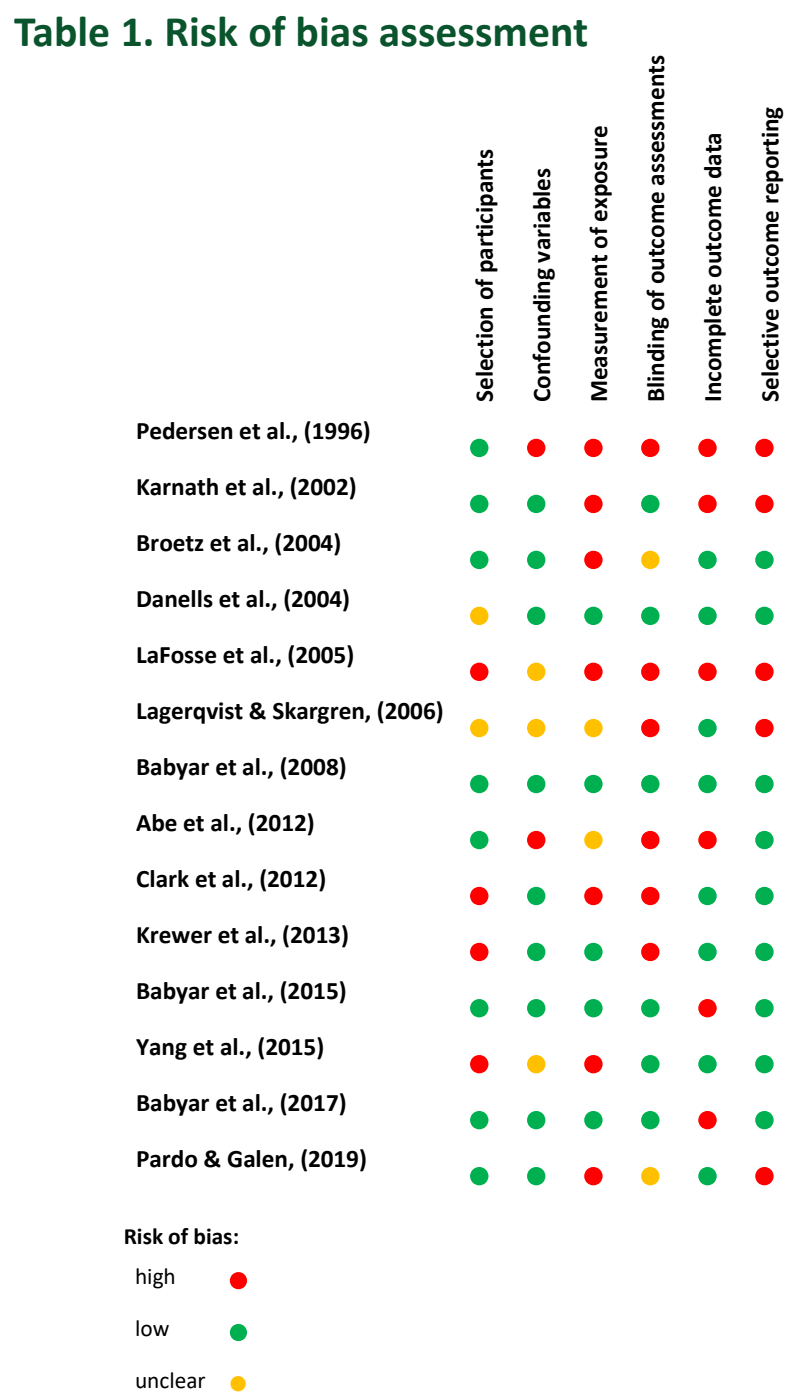

\section{Results}

Ninety-two articles were identified for screening. Fourteen articles met the inclusion criteria. Ten (71\% of articles included) were small case series or cohort studies. The four larger studies reported on cohorts of $52-169$ patients with lateropulsion. Thirteen (93\% of studies) reported on recovery of lateropulsion. Lateropulsion severity can improve with rehabilitation. Right-sided lesions, presence of additional post-stroke impairments, and higher severity of lateropulsion on admission to rehabilitation are associated with delayed recovery from lateropulsion. Ten (71\% of studies) reported on length of stay. Presence of lateropulsion after stroke is associated with requirement for longer rehabilitation length of stay. Nine (64\% of studies) reported on functional outcome. Patients with lateropulsion after stroke may make the same functional improvements in rehabilitation as those without, but over a longer period of time. Six (43\% of studies) reported on discharge destination. There is some disagreement between studies about the association of lateropulsion with discharge destination, but it is likely that when given longer duration of rehabilitation to achieve functional goals, patients with lateropulsion may be discharged home at similar rates to those without lateropulsion.

\section{Conclusions}

The majority of studies investigating these associations used small samples of convenience. Studies used different tools to define and measure lateropulsion and conducted assessments at different timepoints. In assessment of quality, an overall trend for increasing rigor of reporting of studies was noted over time, with lower risk of bias in more recent studies. People with lateropulsion after stroke can achieve similar levels of function and have similar likelihood of returning home as their counterparts without lateropulsion, but require a longer period of rehabilitation to achieve these outcomes. This finding has implications for resourcing rehabilitation services, and the potential to reduce long term cost and burden of care needs cannot be underestimated. However, limitations including variability in assessment tools and time points for evaluation in current literature, as well as small sample sizes reported, mean that caution should be taken when interpreting these data. Further studies including a large cohort of people with lateropulsion after stroke, controlling for stroke severity, are recommended to explore the association of lateropulsion with functional outcome, rehabilitation length of stay and discharge destination.

Acknowledgements

Australian Government Research Training Program Scholarship

Physiotherapy Department, Osborne Park Hospita Stroke Services, Osborne Park Hospital

References

Available on request

Correspondence

Jessica Nolan: jess.nolan@health.wa.gov.au 